\title{
A Comparative Study of Metaheuristic Algorithms for Wave Energy Converter Power Take-Off Optimisation: A Case Study for Eastern Australia
}

\author{
Erfan Amini $^{1}{ }^{(\mathbb{D}}$, Danial Golbaz $^{1}$, Rojin Asadi ${ }^{1}$ (D), Mahdieh Nasiri ${ }^{2}$, Oğuzhan Ceylan ${ }^{3}$, Meysam Majidi Nezhad ${ }^{4}$ \\ and Mehdi Neshat ${ }^{5, *}$
}

check for

updates

Citation: Amini, E.; Golbaz, D.; Asadi, R.; Nasiri, M.; Ceylan, O.; Majidi Nezhad, M.; Neshat, M. A Comparative Study of Metaheuristic Algorithms for Wave Energy Converter Power Take-Off Optimisation: A Case Study for Eastern Australia. J. Mar. Sci. Eng 2021, 9, 490. https://doi.org/ $10.3390 /$ jmse 9050490

Academic Editor: Kyong-hwan Kim

Received: 29 March 2021

Accepted: 27 April 2021

Published: 1 May 2021

Publisher's Note: MDPI stays neutral with regard to jurisdictional claims in published maps and institutional affiliations.

Copyright: (c) 2021 by the authors. Licensee MDPI, Basel, Switzerland. This article is an open access article distributed under the terms and conditions of the Creative Commons Attribution (CC BY) license (https:/ / creativecommons.org/licenses/by/ $4.0 /)$.
1 School of Civil Engineering, College of Engineering, University of Tehran, Tehran 13145, Iran; erfan.amini@ut.ac.ir (E.A.); Dgolbaz@ut.ac.ir (D.G.); rojinasadi@ut.ac.ir (R.A.)

2 School of Mechanical Engineering, Iran University of Science and Technology, Tehran 13114, Iran; mahdie_nasiri@alumni.iust.ac.ir

3 Management Information Systems Department, Kadir Has University, Istanbul 34083, Turkey; oguzhan.ceylan@khas.edu.tr

4 Department of Astronautics, Electrical and Energy Engineering (DIAEE), Sapienza University of Rome, 00184 Rome, Italy; meysam.majidinezhad@uniroma1.it

5 School of Computer Science, The University of Adelaide, Adelaide 5005, Australia

* Correspondence: mehdi.neshat@adelaide.edu.au; Tel.:+610883134729

\begin{abstract}
One of the most encouraging sorts of renewable energy is ocean wave energy. In spite of a large number of investigations in this field during the last decade, wave energy technologies are recognised as neither mature nor broadly commercialised compared to other renewable energy technologies. In this paper, we develop and optimise Power Take-off (PTO) configurations of a well-known wave energy converter (WEC) called a point absorber. This WEC is a fully submerged buoy with three tethers, which was proposed and developed by Carnegie Clean Energy Company in Australia. Optimising the WEC's PTO parameters is a challenging engineering problem due to the high dimensionality and complexity of the search space. This research compares the performance of five state-of-the-art metaheuristics (including Covariance Matrix Adaptation Evolution Strategy, Gray Wolf optimiser, Harris Hawks optimisation, and Grasshopper Optimisation Algorithm) based on the real wave scenario in Sydney sea state. The experimental achievements show that the Multiverse optimisation (MVO) algorithm performs better than the other metaheuristics applied in this work.
\end{abstract}

Keywords: wave energy converter; power take-off system; optimisation; evolutionary algorithms; metaheuristic

\section{Introduction}

The recent uprising demand for more energy resources is imposing huge pressure on energy supply industries [1]. The most sustainable solution to offset the current depletion of fossil energy resources is using renewable energy technologies. Marine wave energy has been considered as a reliable source for coastal demands since 1799 [2]. Thus, its development has occurred vastly-compared to the other resources-due to the following benefits: (i) It is another source of sustainable energy contributing to the mix of energy resources that leads to more diversity and attraction for coastal cities and suppliers [3]. (ii) Wave energy could be exploited offshore and does not need any land, resulting in a lower cost of in-land site selection and reducing unfavourable visual impact [4]. (iii) By considering the best layout and offshore site location, the permanent generation of energy will be accessible (compared to solar energy, for instance, which is time-dependent) [5]. By taking all the mentioned reasons into account, recent studies have focused on enhancing the performance of wave energy technologies by developing optimisation-based solutions in the section of geometric design, PTO parameters, and layout. The second division proved 
to have a crucial role in energy transmission and exploitation in electricity networks [6]. While the energy sector is developing different technologies in this area, some challenges still need to be dealt with, such as design limitations [7], operation and maintenance difficulties in offshore area, power take-off efficiency under heavy currents, etc. [8]. Hence, it is not far-fetched to assume that optimal solutions are always required to achieve better performance. A summary of recent studies has been classified in Table 1 .

Table 1. A brief survey of the recent investigation on the optimisation of the layout of WECs, parameters, and design of PTO.

\begin{tabular}{|c|c|c|c|}
\hline Objective & WECs Mode & Approach & Reference \\
\hline both shape and PTOs & submerged & Experimental observations & [9] \\
\hline both shape and PTOs & fully-submerged & Hybrid evolutionary algorithm & [10] \\
\hline both shape and PTOs & heaving converters & GA and Evolutionary & [11] \\
\hline both shape and Layout & cylindrical heaving WECs & GA & [12] \\
\hline both layout and PTOs & fully-submerged & Hybrid evolutionary algorithm & [13] \\
\hline both layout and PTOs & fully-submerged & metaheuristics & [14] \\
\hline both layout and PTOs & submerged & hybrid GA & [15] \\
\hline both layout and PTOs & fully-submerged & Cooperative optimisation method & {$[16]$} \\
\hline Layout & wave surge converter (oscillating) & Numerical and GWO & {$[17,18]$} \\
\hline PTOs & wave surge converter (oscillating) & GA & {$[19,20]$} \\
\hline PTOs & water column-based (oscillating) & Water cycle algorithm & [21] \\
\hline PTOs & hinged-type WECs & Experimental observations & [22] \\
\hline PTOs & oscillating wave surge converter & both GA and neural model & [23] \\
\hline PTOs & freely floating & EAs & {$[24]$} \\
\hline PTOs & submerged & Hidden GA & [25] \\
\hline Shape & sloped-motion WEC & Heuristic optimisation & [26] \\
\hline Shape & hinge-barge WEC & gradient-based method & [27] \\
\hline Shape & fully-submerged & both PSO and GA & {$[28]$} \\
\hline Shape & submerged flat plate & GA & [29] \\
\hline Shape & submerged & GA & [30] \\
\hline
\end{tabular}

In the following, this article is classified into five sections. We describe a summary of the hydrodynamic interaction model and wave resource, and present the computation of the produced power in Section 2. Section 3 shows the optimisation strategies and manifests the details of the Multiverse optimisation (MVO) algorithm. In Section 4, we discuss the power take-off optimisation results in terms of efficiency and produced power output. Finally, in Section 5, we summarise the major findings of this comparative study, and give our suggestion for future studies.

\section{Model Setup}

In this section, we introduce the wave scenario in the selected site on the eastern coasts of Australia. Then, we describe the geometry of the converter and analyse the governing equation of the motion of the converter's body. Finally, by considering all implied forces into account, the power output of the WEC is numerically simulated.

\subsection{Wave Resource}

In this study, a site on the eastern coast of Australia (Sydney) is investigated according to the 2016 real wave dataset [31]. One of the main ways to represent wave climate is the wave rose, which is a graphical demonstration of significant wave height over its various directions [16].

For instance, the dominant sea state specifies Sydney with a peak period $\left(T_{p}\right)$ around $9 \mathrm{~s}$ and a Significant Wave Height $\left(H_{s}\right)$ of $2 \mathrm{~m}$; the Pierson-Moskowitz wave spectrum is helpful for identifying the probability distribution of the wave frequency $\left(f_{w}\right)$. In fact, the zone of plausible incident wave angle $(\beta)$ at Sydney is 180 . Due to the diversity in directional distributions and probable cumulative energy, wave regimes may have differences with one another. Therefore, Sydney has been chosen to assess the performance 
of optimisation methods. In this research, a modified spectrum called Bretschnider is used, which needs two parameters $\left(T_{p}\right.$ and $\left.H_{s}\right)$ to model irregular waves. It is worth considering the fetch length and the storm's location together along with wind speed and wind direction as they can change the spectrum on a different level.

$$
\begin{gathered}
S(f)=\frac{H_{m 0}^{2}}{4}\left(1.057 f_{p}\right)^{4} f^{-5} \exp \left[-\frac{5}{4}\left(\frac{f_{p}}{f}\right)^{4}\right] \\
A=\frac{H_{m 0}^{2}}{4}\left(1.057 f_{p}\right)^{4} \approx \frac{5}{16} H_{m 0}^{2} f_{p}^{4}, \\
B=\left(1.057 f_{p}\right)^{4} \approx \frac{5}{4} f_{p}^{4} .
\end{gathered}
$$

In Equation (1), $f_{p}$ and $H_{m 0}$ are peak wave period and significant wave height, respectively.

\subsection{Wave Energy Converter Modelling}

A fully submerged, symmetric and spherical buoy is used as the WEC in this study. To keep the buoy in location with specific tolerances against both severe storm load and normal load conditions, the buoy has equipped with three tethers, each one is connected to a mooring point on the seabed with an angle of 55 degrees $(\alpha)$. Details of the buoy parameters can be seen in Table 2 and Figure 1 demonstrates the buoy perfectly [16].

Table 2. Parameters of the spherical WEC.

\begin{tabular}{ccc}
\hline Parameter & Unit & Value \\
\hline Radius, $a$ & $\mathrm{~m}$ & 5 \\
Water depth & $\mathrm{m}$ & 50 \\
Submersion (top of the buoy) & $\mathrm{m}$ & 8 \\
Volume, V & $\mathrm{m}^{3}$ & 524 \\
\hline
\end{tabular}

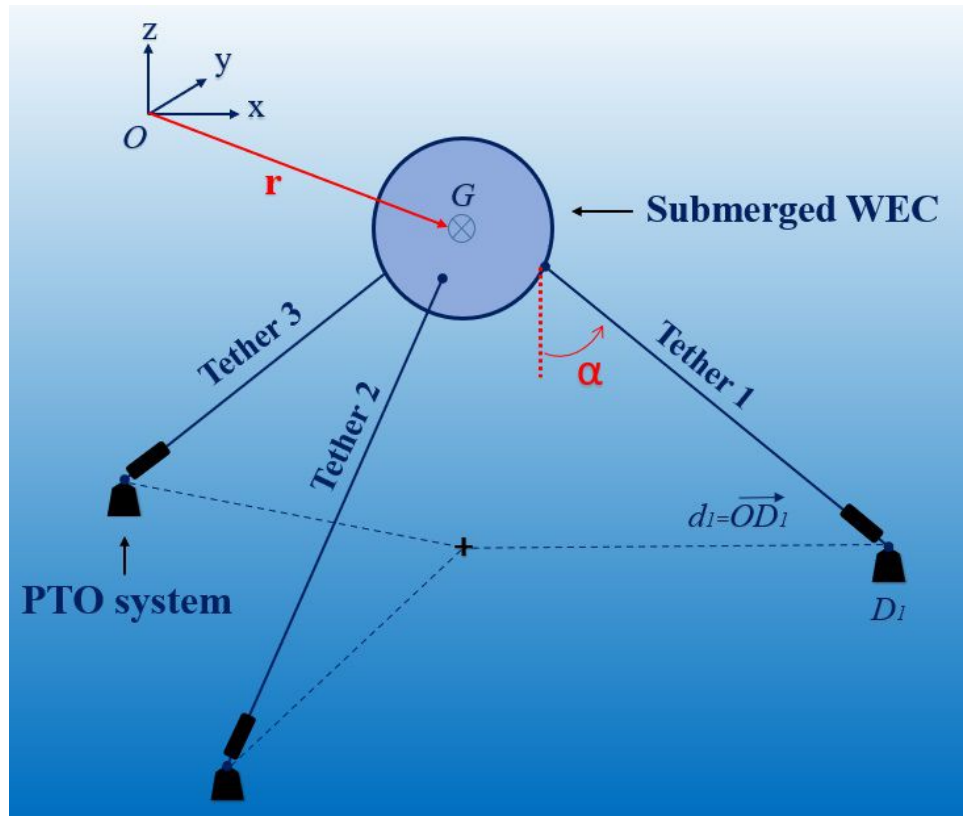

Figure 1. 1Schematic of submerged wave energy converter, including three tethers and PTO system attached to the sea bed.

The Frequency-domain simulation provides a faster approximation, which is applied in this research. This simulation enjoys a significant advantage when it comes to running many model evaluations in designing different scenarios. In the frequency-domain, a linear PTO model is applied. We require modelling of only translational degrees of freedom sway, surge, and heave. The buoy can harness energy from all modes of tethers [16,32]. 
The buoy's three tethers have power take-off mechanisms, which can be modelled as an electric generator or a hydraulic circuit. A PTO system is connected to each tether and the loading force from each of them is assumed to have a linear spring and a damping effect relating to the tether lengthening and rate of change of its lengthening [33].

The fully submerged buoy bears forces that play important roles in numerical modelling. It includes exerted force from the PTO system and excitation force. The excitation forces are felt when the body stands in front of incoming waves, while radiation forces are sensed when the body moves in a steady-state. To calculate the excitation force, incident and scattered waves in hydrodynamic pressure need to be summed up. On the other side, Radiation forces arise on account of body motions, and the radiation resistance, which may be presumed as wave damping force, also has a relation with the average energy exchanging among the WEC's body and sea. This force informs us about the amount of harnessed energy from incoming waves. Another force is added-mass, which is the inertia of water entrained with body motion. It should be considered that the optimal device parameters contingent upon the wave frequency would change relentlessly [4]. The mooring system is designed according to the linear wave theory, considering small motion amplitude compared with tethers' length. The following $S_{i}$ indicates the spatial arrangement of tethers.

$$
S_{i}=r+R n_{i}-d_{i}, \quad i=1 \cdot 3,
$$

where in the reference coordinate frame $O_{x y z}, r$ is the vector of buoy's position and $R$ is the radiation matrix. The position vector of the anchor point of tether $i$ is related to $G$, which is the centre of mass, and $d_{i}$ is the position vector of the anchor point of tether $i$ on the seafloor in $O_{x y z}$. The length of tether can be calculated as

$$
l_{i}=\left\|s_{i}\right\|=\sqrt{s_{i}^{T} s_{i}}, \quad i=1 \ldots 3,
$$

and the change of the length is modelled as $\Delta l_{i}=l_{i}-l_{0}$ [34]. The below equation is used for modelling the PTO force.

$$
F_{p t o, i}=\left(C_{p t o}-D_{p t o} \Delta \dot{l}_{i}-K_{p t o} \Delta l_{i}\right), \quad i=1 \ldots 3,
$$

where $D_{\text {pto }}$ and $K_{\text {pto }}$ are the damping and stiffness matrices, respectively. $C_{p t o}$ is a counteracting force of hydrostatic force, which is obtained from the following equation:

$$
C_{p t o}=-\frac{\left(m_{w}-m_{b}\right) g}{3 \cos \alpha},
$$

where $m_{w}$ is the mass of displaced water, $m_{b}$ is the mass of the buoy, $g$ is the gravitational acceleration constant, and $\alpha$ is assumed to be 55 degrees as proposed in [34]. The buoy velocities can be mapped in a Cartesian coordinate frame considering the change rate of the tether length, which is provided by the inverse kinematic Jacobian in [34]. Other forces including viscous forces and the end stop forces are neglected and kinematics are linearised in this research. Among the discussed forces, the most important one is the excitation force, which is calculated by Equation (6) [16].

$$
\begin{aligned}
\hat{F}_{\text {exc }} & =\left((M+A(\omega)) j \omega+B(\omega)-\frac{K_{p t o}}{\omega} j+D_{p t o}\right) \ddot{X} \\
M & =m I_{3} \\
K_{p t o} & =K_{p t o} I_{3} \\
D_{p t o} & =D_{p t o} I_{3},
\end{aligned}
$$

where $F_{e x c}$ is the frequency-dependent vector of excitation forces, $M$ is the mass matrix, and $I$ is the identity matrix. We use a constant 3 value because there are three degrees of freedom. $\ddot{X}$ is a vector of body acceleration in the surge, heave, and sway directions. 
Added-mass and radiation forces are defined through A and B matrices, respectively. There are three tethers attached to the buoy modelled as an oscillating spring. There are 50 individual $K_{\text {pto }}$ and $D_{\text {pto }}$ parameters; therefore, in each tether, there are 100 individuals, and in total it will be 300 for a buoy. Hydrodynamic reactions can be calculated according to the semianalytical solution described in [35].

$$
P=\frac{1}{4}\left(\hat{F}_{e x c}^{*} \ddot{X}+\ddot{X} \hat{F}_{e x c}\right)-\frac{1}{2} \ddot{X}^{*} B \ddot{X}^{*} .
$$

The power output of the buoy is calculated in an irregular wave frequency domain according to Equation (7) [16]. In this study, the applied wave energy converter simulator was developed and published by Sergiienko on Matlab-R2019b [36].

\section{Optimisation}

In this section, we describe the general formulation of the problem regarding the optimisation viewpoint, then, we briefly introduce optimisation algorithms. Finally, the MVO optimiser has been reviewed as a novel, population-based optimiser in the application of WEC's PTO assessment.

\subsection{Optimisation Formulation}

The applied formulation of the optimisation problem in order to maximise the produced power output of the WEC can be seen in the following:

$$
\text { Power }=\operatorname{argmax}_{K_{p t o}, D_{p t o}} \operatorname{Power}\left(K_{p t o}, D_{p t o}\right),
$$

where Power represents the annual average power generated for given PTO settings of a WEC where the location is fixed: Power Take-off settings incorporating $\overrightarrow{K_{p t o}}=$ $\left\{\left[T_{k_{1}}^{1}, \ldots, T_{k_{50}}^{1}\right],\left[T_{k_{1}}^{2}, \ldots, T_{k_{50}}^{2}\right],\left[T_{k_{1}}^{3}, \ldots, T_{k_{50}}^{3}\right]\right\}$ and $\overrightarrow{D_{p t o}}=\left\{\left[T_{d_{1}}^{1}, \ldots, T_{d_{50}}^{1}\right],\left[T_{d_{1}}^{2}, \ldots, T_{d_{50}}^{2}\right]\right.$, $\left.\left[T_{d_{1}}^{3}, \ldots, T_{d_{50}}^{3}\right]\right\}$, where $T$ is the $i$ th tether of WEC. It is considered that the WEC is installed in the ocean at a depth at $3(\mathrm{~m})$ with a uniform depth of $30(\mathrm{~m})$. The PTO constraints are on the coefficients of spring and damping parameters at $d_{l}=5 \times 10^{4}, d_{u}=4 \times 10^{5}$, and $k_{l}=1, k_{u}=5.5 \times 10^{5}$. If a candidate solution does not satisfy the constraint values, it is recognised as an infeasible solution. In order to handle the boundary PTO constraints, infeasible solutions are limited to the most nearby feasible areas.

\subsection{Optimisation Algorithms}

In this study, we use five metaheuristic swarm optimisation algorithms to achieve the optimal values of power take-off parameters in terms of either damping or stiffness variables. These algorithms have been chosen based on recent reviews of their application in WECs' power take-off performance assessments $[37,38]$. The collected algorithms include the Covariance Matrix Adaptation Evolution Strategy (CMA-ES) [39], Gray Wolf optimiser [40], Harris Hawks optimisation [41], and Grasshopper Optimisation Algorithm [42]. More specifically, we go through the application details of another recently released metaheuristic optimisation algorithm called Multiverse optimiser. All algorithms have been used for 10,000 total evaluations, carried out by 25 search agents at 400 iterations. We replicate each experiment ten times to find the minimum, maximum, mean, and standard deviation for each optimiser in this problem. Table 3 presents the settings of the five employed algorithms. 
Table 3. The settings of optimisation approaches. The maximum evaluation number is $10^{5}$.

\begin{tabular}{ll}
\hline Algorithms & Settings \\
\hline CMA-ES [39] & with the default settings and $n P o p=25 ;$ \\
MVO [43] & with $n P o p=25$, (decreased with a damping ratio $w_{f}=0.99$ \\
GWO [40] & with $n P o p=25$ and the de,$\alpha=2$ (linearly decreased to zero) \\
GOA [42] & with $n P o p=25$, and the default settings \\
HHO [41] & with $n P o p=25$, and the default settings \\
\hline
\end{tabular}

As the application of CMA-ES, GOA, HHO, and GWO algorithms have been studied individually in the previous works $[17,44]$, we will go through details of the state-of-the-art Multiverse optimiser for this problem as follows.

\subsection{Multiverse Optimiser (MVO)}

In a recent released study [43], a promising and novel population-based optimisation algorithm is introduced entitled "Multiverse optimiser (MVO)" that divides the search mode toward the principal two steps: exploration and exploitation. In the MVO method, the main inspiration is related to the theories of black holes and, inversely, white holes to perform the exploitation and exploration processes, respectively. Furthermore, wormholes are effective in order to develop the search abilities as a local search strategy. In spite of what preceded, some distinct concepts are implemented in the MVO including a universe that represents a solution, an individual in the universe corresponds to each variable of the solution, and the inflation rate accords with the value of the fitness function. The main strategies of the MVO algorithm can be seen in the following:

1. The larger values of the inflation rate are regarded as performing a white hole with a higher probability rate than that of a black hole appearing.

2. A universe with a large inflation degree leads to transfer candidates. However, a low inflation rate results in receiving candidates within black and white spirits.

3. Total elements in the universe will stochastically turn around the best candidate within the wormhole, notwithstanding the statistical values of the inflation rate.

In each generation of the MVO approach, a roulette-wheel technique is applied to choose a white hole from the total universes based on the inflation rate. The main objective of this mechanism is to improve candidates transfer in various universes and also to develop exploration abilities. Thus, the MVO scheme assumes that, as follows:

$$
U=\left[\begin{array}{cccc}
x_{1}^{1} & x_{1}^{2} & \ldots & x_{1}^{d} \\
x_{2}^{1} & x_{2}^{2} & \ldots & x_{1}^{d} \\
\vdots & \vdots & & \vdots \\
x_{n}^{1} & x_{n}^{2} & \ldots & x_{n}^{d}
\end{array}\right]
$$

where $n$ is the number of solutions in the population and $d$ is the length of decision variables. Therefore, each solution can be defined as follows:

$$
x_{i}^{j}= \begin{cases}x_{k}^{j} & r_{1}<N I\left(U_{i}\right), \\ x_{i}^{j} & r_{1} \geq N I\left(U_{i}\right) .\end{cases}
$$

In Equation (10), $r_{1}$ shows a random number in the interval of $[0,1], x_{i}^{j}$ symbolises the $j$ th variable of $i$ th universe, where $x_{k}^{j}$ shows the $j$ th variable of $k$ th universe chosen by a roulette-wheel selection algorithm. Meanwhile, $U_{i}$ explains the $i$ th universe and NI(Ui) 
denotes the rate of normalised inflation of the $i$ th universe, so updating the new location of the candidates is as follows:

$$
x_{i}^{j}=\left\{\begin{array}{l}
x_{j}+\mathrm{TDR} \times\left(u b_{j}-l b_{j}\right) \times r_{4}+l b_{j} \\
\longrightarrow \text { if }\left(r_{3}<0.5\right) \&\left(r_{2}<\mathrm{WEP}\right) \\
x_{j}-\mathrm{TDR} \times\left(u b_{j}-l b_{j}\right) \times r_{4}+l b_{j} \\
\longrightarrow \text { if }\left(r_{3} \geq 0.5\right) \&\left(r_{2}<\mathrm{WEP}\right), \\
x_{i}^{j} \quad \text { if }\left(r_{2} \geq \mathrm{WEP}\right),
\end{array}\right.
$$

where $r_{2}, r_{3}$, and $r_{4}$ are generated random numbers within [0, 1], $x_{i}^{j}$ mentions the $j$ th variable of $i$ th universe, and $x_{j}$ intimates the $j$ th variable of the best universe developed so far. Both TDR and WEP are applied as the coefficients, $l b_{j}$ determines the lower bound of $j$ th variable and $u b_{j}$ denotes the upper bound of $j$ th variable. In the MVO algorithm, there are two significant coefficients including wormhole existence probability (WEP) and travelling distance rate (TDR). Both coefficients are developed as adaptive formulas as follows:

$$
\mathrm{WEP}=\min +\text { iter } *\left(\frac{\max -\min }{M a x_{i t e r}}\right),
$$

where min and max are constant values at 0.2 and 1 , respectively. In the original MVO, iter is the current iteration and Maxiter is the maximum number of iterations.

$$
\mathrm{TDR}=1-\frac{i t e r^{1 / \rho}}{\operatorname{Max}_{i t e r}^{1 / \rho}} .
$$

Finally, $\rho$ implies the exploitation accuracy beyond the iterations, which is chosen as 6 in the original MVO paper. Where the $\rho$ value progresses higher, it is likely to receive more advanced and precise exploitation. Moreover, the Quick-sort algorithm is proposed to sort the universe after each iteration. This process facilitates finding optimal answers in this optimisation problem in terms of power take-off variables.

\section{Experimental Optimisation Results}

In this section, the experimental results of optimising parameters are presented. The output records of the model revealed the convergence curve of the converter's power output to the maximum value. Then, the minimum, maximum, mean value, and standard deviation of the experiments have been calculated. Furthermore, the overall trend of PTO coefficient changes has been recorded, followed by statistical analysis of achieved results based on the PTO's coefficient configuration. As mentioned in Section 3.2, each experiment comprises $10^{5}$ evaluation numbers. The numerical analysis has been carried out using five different metaheuristic promising optimisation algorithms, including MVO, CMA-ES, GWO, GOA, and HHO. To begin with, Figure 2 tracks the convergence of WEC's power output to its maximum amount using five above mentioned optimisation algorithms over $10^{5}$ iterations.

By looking at Figure 2, we can perceive the competence of each algorithm to optimise this parametric problem. As it can be easily seen, the Multiverse optimiser succeeds other algorithms to extract higher power. According to the search process attributes of the HHO algorithm, it seems to be stuck in local optima, thereby losing its chance to perform an exhaustive search throughout the whole search space. Second to the MVO, the CMA-ES approach achieves the best results, followed by the other three algorithms by a non-negligible distance. In brief, both MVO and CMA-ES were observed to be the most productive methods of optimising in this problem. 


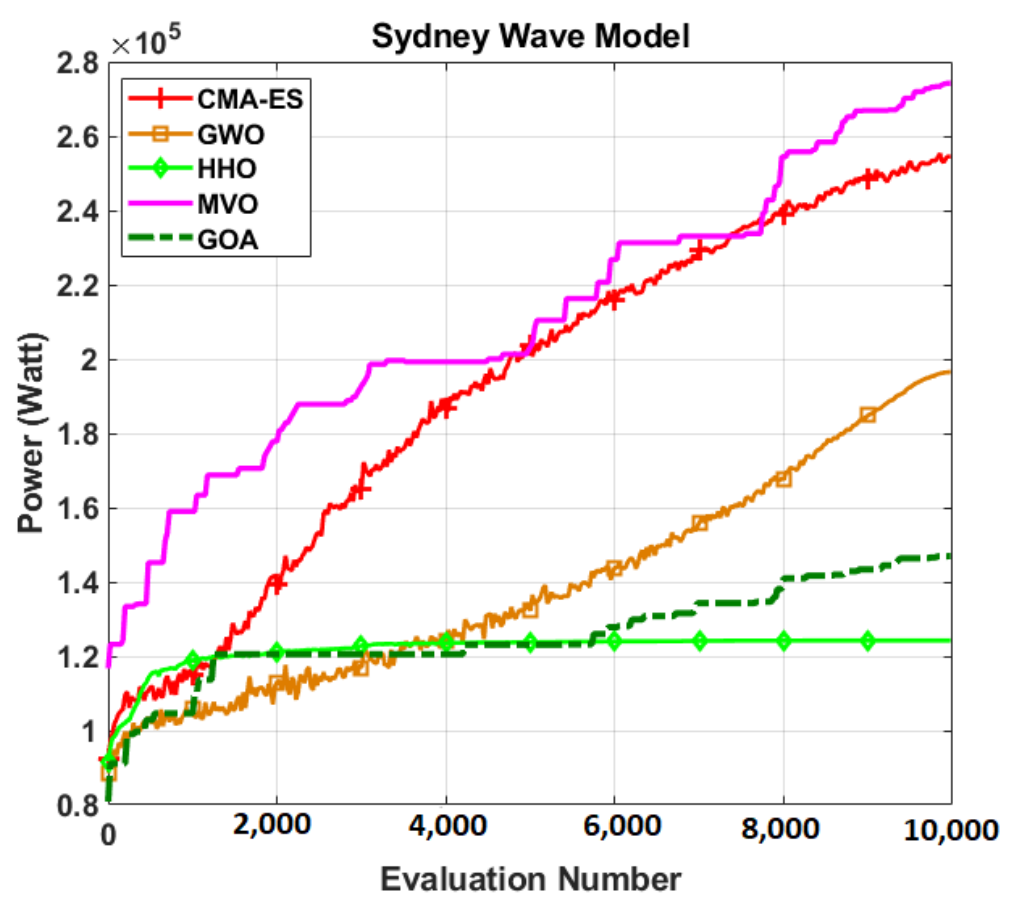

Figure 2. A comparison of convergence curve of five metaheuristics to optimise the configuration of power take-off parameters.

In order to examine the performance of studied methods to find the optimal number of PTO coefficients, we analyse the trajectory of damping and spring coefficients for each of the three PTO systems in three different frequency samples-including 1, 25, and 50-in Figure 3.

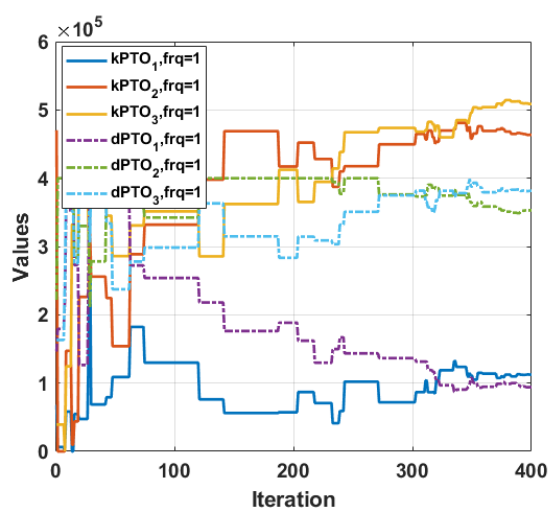

(a)

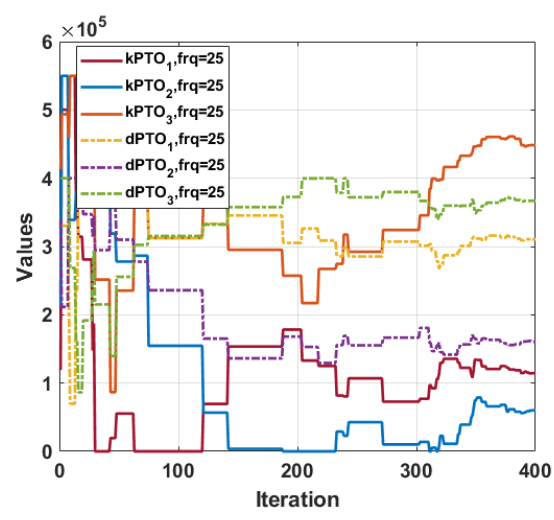

(b)

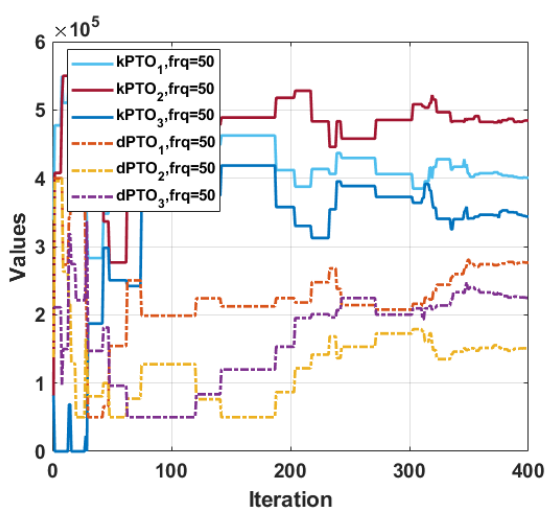

(c)

Figure 3. The trajectory of damping and spring coefficient optimisation from three PTO systems in the three different frequency samples: (a) frequency sample 1, (b) frequency sample 25, and (c) frequency sample 50.

Looking at Figure 3, we can observe that the parameter coefficients for each PTO stiffness of the damping system change nonmonotonically over the course of iterations as its frequency changes. Moreover, high chaotic fluctuations are observed at the beginning of the search process in all frequencies, specifying the exploration phase of the searching process.

Having a closer look at variables, most damping coefficients tend to reach higher values than stiffness coefficients in lower frequencies, like 1 and 25. On the other hand, this trend is vice versa in the 50th frequency. Having said that, the maximum changes from the initial amount are experienced by the stiffness coefficient. This leads us to the fact that the 
objective function is affected by this variable more than those related to the damping part of the PTO system.

Figure 4 presents the box-and-whisker plot for the power output of the best solution per run for all search metaheuristic algorithms for the Sydney wave scenario on the eastern Australian coasts.

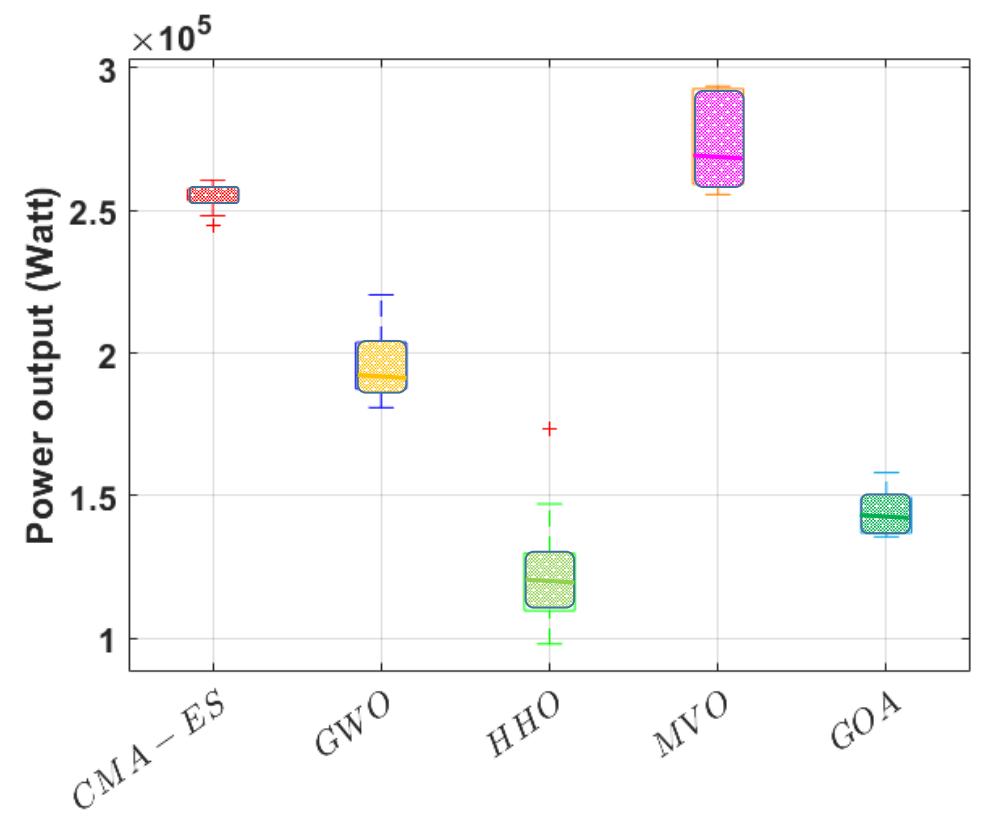

Figure 4. Statistical results of five optimisation algorithms to optimise the configuration of power take-off parameters.

Considering Figure 4, a comparative-statistical analysis can be found. The best mean power output of the WEC is observed using the MVO method. It is followed by the CMA-ES, GWO, GOA, and HHO methods, respectively. Furthermore, the dispersion of the achieved data over the mean value is high in the GWO and HHO methods compared to CMA-ES and GOA. Overall, as the power output is just under $300 \mathrm{~kW}$ considering MVO, this method is found to be the most compatible algorithm for this optimisation problem.

Finally, Table 4 summarises the mean, minimum, maximum, and standard deviation of each algorithm's results over 10 runs.

Table 4. Experimental optimisation results of five applied optimisation algorithms.

\begin{tabular}{cccccc}
\hline Method & CMA-ES & GWO & HHO & MVO & GOA \\
\hline Mean & $2.545 \times 10^{5}$ & $1.965 \times 10^{5}$ & $1.242 \times 10^{5}$ & $2.725 \times 10^{5}$ & $1.451 \times 10^{5}$ \\
Min & $2.446 \times 10^{5}$ & $1.809 \times 10^{5}$ & $9.799 \times 10^{4}$ & $2.556 \times 10^{5}$ & $1.356 \times 10^{5}$ \\
Max & $2.606 \times 10^{5}$ & $2.204 \times 10^{5}$ & $1.733 \times 10^{5}$ & $2.935 \times 10^{5}$ & $1.581 \times 10^{5}$ \\
STD & $5.021 \times 10^{3}$ & $1.220 \times 10^{4}$ & $2.285 \times 10^{4}$ & $1.574 \times 10^{4}$ & $7.434 \times 10^{3}$ \\
\hline
\end{tabular}

Table 4 reveals that the maximum power output of the converter can be produced by letting the MVO algorithm use optimum PTO coefficients. This maximum result is followed by the results of CMA-ES, GWO, HHO, and GOA, respectively. Regarding the mean and minimum amount of power outputs, these trends are found to be repeated. As for the standard deviation of the results, we can sort the algorithms in order as $\mathrm{HHO}, \mathrm{MVO}$, GWO, GOA, and CMA-ES. Additionally, the convergence of the results is more precise in HHO and MVO while, on the other hand, results from GWO, GOA, and CMA-ES are much more spread out. 


\section{Conclusions}

In this paper, we developed a comparative optimisation framework in order to evaluate and optimise the Power Take-off (PTO) settings of a fully-submerged three-tether wave energy converter (WEC). This WEC was introduced and developed by Carnegie Clean Energy Company in Australia.

Due to a large-scale dimension of PTO parameters and complex interactions among three tethers, optimising the WEC's PTO parameters is a challenging engineering problem. In this way, we developed and compared the effectiveness of five state-of-the-art metaheuristic algorithms on the Sydney wave scenario, Australia. The preliminary results confirm that the Multiverse optimisation (MVO) algorithm can considerably outperform the other four optimisation algorithms applied in this paper. Second to the MVO, the highest mean value and maximum value of the WEC's power output were achieved using CMA-ES, GWO, GOA, and HHO, respectively. As another concluding remark of this study, it worth mentioning that the stiffness coefficients proved to have more effect on objective functions than damping coefficient in the studied converter's power take-off system.

In future work, we will develop a more comprehensive comparative framework to analyse the performance of a wide range of Genetic, Evolutionary, and Swarm optimisation algorithms in order to optimise the PTO settings. Furthermore, developing and assessing the impact of hybridisation, alternating, and cooperative optimisation techniques can be considered.

Author Contributions: Conceptualization, E.A. and M.N. (Mehdi Neshat); data curation, D.G., E.A., M.N. (Mehdi Neshat), and M.M.N.; formal analysis, E.A., D.G., and R.A.; investigation, E.A., D.G., R.A., M.N. (Mahdieh Nasiri), and O.C.; methodology, E.A., M.N. (Mehdi Neshat), and M.M.N.; resources, E.A. and O.C.; validation, E.A., D.G., R.A., and M.M.N.; visualization, E.A. and M.N. (Mahdieh Nasiri); writing—original draft, E.A., D.G., R.A., and M.N. (Mehdi Neshat); writingreview \& editing, M.N. (Mehdi Neshat), M.M.N. and O.C.; supervision, M.N. (Mehdi Neshat); correspondence, M.N. (Mehdi Neshat). All authors have read and agreed to the published version of the manuscript.

Funding: This research received no external funding.

Institutional Review Board Statement: Ethical review and approval were waived for this study, due to studies not involving humans or animals.

Conflicts of Interest: The authors declare no conflict of interest.

\begin{tabular}{|c|c|}
\hline \multicolumn{2}{|c|}{ Abbreviations } \\
\hline \multicolumn{2}{|c|}{ The following abbreviations are used in this manuscript: } \\
\hline \\
\hline PTO & Power Take-off system \\
\hline GWO & Gray Wolf Optimiser \\
\hline MVO & Multiverse Optimiser \\
\hline CMA-ES & Covariance Matrix Adaptation Evolution Strategy \\
\hline $\mathrm{HHO}$ & Harris Hawks Optimisation \\
\hline GOA & Grasshopper Optimisation Algorithm \\
\hline
\end{tabular}

\section{References}

1. Babarit, A. Energy Resource and Markets. In Ocean Wave Energy Conversion; Elsevier Science: Amsterdam, The Netherlands, 2018; Volume 1, pp. 37-42.

2. Aderinto, T.; Li, H. Ocean Wave Energy Converters: Status and Challenges. Energies 2018, 11, 1250. [CrossRef]

3. Castellani, B.; Morini, E.; Nastasi, B.; Nicolini, A.; Ross, F. Small-Scale Compressed Air Energy Storage Application for Renewable Energy Integration in a Listed Building. Energies 2018, 11, 1921. [CrossRef]

4. Pecher, A.; Kofoed, J.P. Handbook of Ocean Wave Energy; Ocean Engineering \& Oceanography; Springer International Publishing: Berlin, Germany, 2017. [CrossRef]

5. Mazzoni, S.; Ooi, S.; Nastasi, B.; Romagnoli, A. Energy storage technologies as techno-economic parameters for master-planning and optimal dispatch in smart multi energy systems. Appl. Energy 2019, 254, 113682. [CrossRef] 
6. Senol, K.; Raessi, M. Enhancing power extraction in bottom-hinged flap-type wave energy converters through advanced power take-off techniques. Ocean. Eng. 2019, 11, 2-22. [CrossRef]

7. Amini, E. Locating and Evaluating the Oscillating Surge Wave Energy Converter Using Grey Wolf Optimizer Algorithm and WEC-Sim Toolbox. Ph.D. Thesis, Tehran University, Tehran, Iran, 2019.

8. Giassi, M. Optimization of Point Absorber Wave Energy Parks. Ph.D. Thesis, Uppsala University, Uppsala, Sweden, 2019.

9. Castro, F.A.; Chiang, L.E. Design optimization and experimental validation of a two-body Wave Energy Converter with adjustable Power Take-Off parameters. Energy Sustain. Dev. 2020, 56, 19-32. [CrossRef]

10. Sergiienko, N.Y.; Neshat, M.; da Silva, L.S.; Alexander, B.; Wagner, M. Design optimisation of a multi-mode wave energy converter. arXiv 2020, arXiv:2001.08966.

11. Bonovas, M.I.; Anagnostopoulos, I.S. Modelling of operation and optimum design of a wave power take-off system with energy storage. Renew. Energy 2020, 147, 502-514. [CrossRef]

12. Lyu, J.; Abdelkhalik, O.; Gauchia, L. Optimization of dimensions and layout of an array of wave energy converters. Ocean. Eng. 2019, 192, 106543. [CrossRef]

13. Neshat, M.; Alexander, B.; Sergiienko, N.Y.; Wagner, M. A Hybrid Evolutionary Algorithm Framework for Optimising Power Take off and Placements of Wave Energy Converters. In Proceedings of the Genetic and Evolutionary Computation Conference, GECCO '19, Prague, Czech Republic, 13-17 July 2019; Association for Computing Machinery: New York, NY, USA, 2019; pp. 1293-1301. [CrossRef]

14. Vatchavayi, S.R. Heuristic Optimization of Wave Energy Converter Arrays. Ph.D. Thesis, University of Minnesota, Minneapolis, MN, USA, 2019.

15. Giassi, M.; Göteman, M. Layout design of wave energy parks by a genetic algorithm. Ocean Eng. 2018, 154, 252-261. [CrossRef]

16. Neshat, M.; Alexander, B.; Wagner, M. A hybrid cooperative co-evolution algorithm framework for optimising power take off and placements of wave energy converters. Inf. Sci. 2020, 534, 218-244. [CrossRef]

17. Amini, E.; Naeeni, S.T.O.; Ghaderi, P. Investigating Wave Energy Potential in Southern Coasts of the Caspian Sea and Evaluating the Application of Gray Wolf Optimizer Algorithm. arXiv 2019, arXiv:1912.13201.

18. Amini, E.; Golbaz, D.; Amini, F.; Majidinezhad, M.; Neshat, M.; Garcia, D.A. Performance investigation on the different wave energy converters layouts in real wave scenarios. arXiv 2020, arXiv:2007.14012.

19. Calvário, M.; Gaspar, J.; Kamarlouei, M.; Hallak, T.; Soares, C.G. Oil-hydraulic power take-off concept for an oscillating wave surge converter. Renew. Energy 2020. [CrossRef]

20. Jusoh, M.; Ibrahim, M.; Daud, M.; Yusop, Z.; Albani, A.; Rahman, S.; Mohad, S. Parameters estimation of hydraulic power take-off system for wave energy conversion system using genetic algorithm. EEES 2020, 463, 012129.

21. M'zoughi, F.; Bouallègue, S.; Garrido, A.J.; Garrido, I.; Ayadi, M. Water cycle algorithm-based airflow control for oscillating water column-based wave energy converters. Proc. Inst. Mech. Eng. Part I J. Syst. Control. Eng. 2020, 234, 118-133. [CrossRef]

22. Chen, M. Hydrodynamic Analysis and Optimization of a Hinged-Type Wave Energy Converter-SeaWEED. Ph.D. Thesis, Memorial University of Newfoundland, St. John's, NL, Canada, 2020.

23. Liu, Z.; Wang, Y.; Hua, X. Prediction and optimization of oscillating wave surge converter using machine learning techniques. Energy Convers. Manag. 2020, 210, 112677. [CrossRef]

24. Jabrali, A.; Khatyr, R.; Naciri, J.K. Viscous effects and energy recovery optimization for freely floating and bottom fixed wave energy converters. Int. J. Renew. Energy Res. 2019, 9, 290-300.

25. Ossama, A.; Shadi, D. Optimization of nonlinear wave energy converters Ocean Eng. 2018, 162, $187-195$.

26. Rodríguez, C.A.; Rosa-Santos, P.; Taveira-Pinto, F. Hydrodynamic optimization of the geometry of a sloped-motion wave energy converter. Ocean. Eng. 2020, 199, 107046. [CrossRef]

27. Wang, L.; Ringwood, J.V. Geometric optimization of a hinge-barge wave energy converter. In Proceedings of the 13th European Wave and Tidal Energy Conference, Napoli, Italy, 1-6 September 2019; p. 1389.

28. Faraggiana, E.; Masters, I.; Chapman, J. Design of an optimization scheme for the WaveSub array. In Advances in Renezable Energies Offshore: Proceedings of the 3rd International Conference on Renewable Energies Offshore (RENEW), Lisbon, Portugal, 8-10 October 2018; Soares, G., Ed.; Taylor \& Francis Group: London, UK, 2019; pp. 633-638.

29. Esmaeilzadeh, S.; Alam, M.R. Shape optimization of wave energy converters for broadband directional incident waves. Ocean. Eng. 2019, 174, 186-200. [CrossRef]

30. Kelly, M.; Alam, M.R. Shape Optimization of a Submerged Pressure Differential Wave Energy Converter for Load Reductions. In Proceedings of the International Conference on Offshore Mechanics and Arctic Engineering, Glasgow, Scotland, UK, 11 November 2019; American Society of Mechanical Engineers: New York, NY, USA, 2019; Volume 58899, p. V010T09A030.

31. Australian Wave Energy Atlas. 2017. Available online: https://arena.gov.au/projects/australian-wave-energy-atlas/ (accessed on 24 October 2017).

32. Sergiienko, N.; Cazzolato, B.; Arjomandi, M.; Ding, B.; da Silva, L. Considerations on the control design for a three-tether wave energy converter. Ocean. Eng. 2019, 183, 469-477. [CrossRef]

33. Scruggs, J.T.; Lattanzio, S.M.; Taflanidis, A.A.; Cassidy, I.L. Optimal causal control of a wave energy converter in a random sea. Appl. Ocean. Res. 2013, 42,1-15. [CrossRef] 
34. Sergiienko, N.Y.; Cazzolato, B.S.; Ding, B.; Arjomandi, M. Three-tether axisymmetric wave energy converter: Estimation of energy delivery. In Proceedings of the 3rd Asian Wave and Tidal Energy Conference, Singapore, 25-27 October 2016; Volume 1, pp. 163-171.

35. Wu, G. Radiation and diffraction by a submerged sphere advancing in water waves of finite depth. Proc. R. Soc. Lond. Ser. A Math. Phys. Sci. 1995, 448, 29-54.

36. Sergiienko, N. Three-Tether Wave Energy Converter-Simulation Tool. MATLAB Central File Exchange. Available online: https: //www.mathworks.com/matlabcentral/ fileexchange/74424-three-tether-wave-energy-converter-simulation-tool (accessed on 14 April 2021).

37. Neshat, M.; Alexander, B.; Wagner, M.; Xia, Y. A detailed comparison of meta-heuristic methods for optimising wave energy converter placements. In Proceedings of the Genetic and Evolutionary Computation Conference, Kyoto, Japan, 15-19 July 2018; pp. 1318-1325.

38. Meng, Z.; Li, G.; Wang, X.; Sait, S.M.; Yıldı, A.R. A comparative study of metaheuristic algorithms for reliability-based design optimization problems. Arch. Comput. Methods Eng. 2020, 28, 1853-1869. [CrossRef]

39. Hansen, N. The CMA evolution strategy: A comparing review. Towards New Evol. Comput. 2006, 192, 75-102.

40. Mirjalili, S.; Mirjalili, S.M.; Lewis, A. Grey wolf optimizer. Adv. Eng. Softw. 2014, 69, 46-61. [CrossRef]

41. Heidari, A.A.; Mirjalili, S.; Faris, H.; Aljarah, I.; Mafarja, M.; Chen, H. Harris hawks optimization: Algorithm and applications. Future Gener. Comput. Syst. 2019, 97, 849-872. [CrossRef]

42. Saremi, S.; Mirjalili, S.; Lewis, A. Grasshopper optimisation algorithm: theory and application. Adv. Eng. Softw. 2017, 105, 30-47. [CrossRef]

43. Mirjalili, S.; Mirjalili, S.M.; Hatamlou, A. Multi-verse optimizer: a nature-inspired algorithm for global optimization. Neural Comput. Appl. 2016, 27, 495-513. [CrossRef]

44. Neshat, M.; Sergiienko, N.Y.; Amini, E.; Majidi Nezhad, M.; Astiaso Garcia, D.; Alexander, B.; Wagner, M. A New Bi-Level Optimisation Framework for Optimising a Multi-Mode Wave Energy Converter Design: A Case Study for the Marettimo Island, Mediterranean Sea. Energies 2020, 13, 5498. [CrossRef] 